\title{
The Kiki-Bouba paradigm : where senses meet and greet
}

\author{
Aditya Shukla \\ Cognitive Psychologist, The OWL, Pune. \\ E-mail-adityashukla77@gmail.com
}

\begin{abstract}
Humans have the ability to think in abstract ways. Experiments over the last 90 years have shown that stimuli from the external world can be evaluated on an abstract spectrum with 'Kiki' on one end and 'Bouba' on the other. People are in concordance with each other with respect to calling a jagged-edgysharp bordered two dimensional shape 'Kiki' and a curvy-smooth-round two dimensional shape 'Bouba'.. The proclivity of this correspondence is ubiquitous. Moreover, the Kiki-Bouba phenomenon seems to represent a non-arbitrary abstract connection between 2 or more stimuli. Studies have shown that crossmodal associations between and withinaudioception, opthalmoception, tactioception and gustatoception can be demonstrated by using Kiki-Bouba as a cognitive 'convergence point'. This review includes a critical assessment of the methods, findings, limitations, plausible explanations and future directions involving the Kiki-Bouba effect. Applications include creatingtreatments and training methods to compensate for poor abstract thinking abilities caused by disorders like schizophrenia and autism, for example. Continuing research in this area would help building a universal model of consciousness that fully incorporates cross-sensory perception at the biological and cognitive levels.
\end{abstract}

Key words:Kiki-Bouba, crossmodal correspondence, multisensory perception, abstraction, ideasthesia, symbolism, schizophrenia, autism.

(Paper received $-1^{\text {st }}$ September 2016, Review completed $-10^{\text {th }}$ September 2016, Accepted $-12^{\text {th }}$ September 2016)

\section{INTRODUCTION}

We often describe objects in the environment in complex ways. These descriptions contain analogies, metaphors, emotional effect and structural and functional details about the objects. In 1929, Wolfgang Kohler demonstrated, for the first time, an inherent tendency in people to match certain sounds to shapes. He showed that people non-arbitrarily matched the non-sense word Takete(later Kiki)to a jagged star like shape and Baluma(later Bouba)to around bulbous shape [1-2]. Cross-modal correspondence typicallyrefers to input from one sensory organ tethering and associating with a stimuli feature from another sensory organ. Theaudio-visual correspondence of Kiki-Bouba is the sound of Kiki matching the shape of Kiki, which is the jagged star shape, for example. In years that followed, extensive research, especially after the year 2000 shows that this phenomenon can be demonstrated with virtually any type of a stimuli [familiar and unfamiliar], or even a single feature of a stimuli across any sensory modality. Now, ample cross-modal and multi-sensory perception evidence has accumulated that verifies this universal phenomenon- now dubbed the 'Kiki-Bouba effect' [3-4]. For example, drum cymbals typically sound Kiki; dark chocolate tastes Kiki, bright lights appear Kiki; while a flute sounds Bouba, milk chocolate tastes Bouba and dim lights appear Bouba. An astonishing accuracy of over $90-95 \%$ is seen in neurologically normal populations, but in autistic people, the accuracy is only slightly better than that expected by chance. The accuracy is measured based on how many people agree on which word matches with which shape. In this case $90 \%$ of people agree that Kiki represents the jagged shape and Bouba represents the bulbous shape as shown in Figure 1[5]. 


\section{STRUCTURE AND PURPOSE OF THE REVIEW PAPER}

This review begins with an explanation of a typical 'Kiki-Bouba' experiment and discusses its testing paradigm. As literature adopting this paradigm has grown enormously with respect to different types of stimuli across multiple sensory modalities, a systematic categorical summary is provided highlighting important, novel and influential findings. This essay includes a critical review of studies employing the Kiki-Bouba paradigm and resultant cross-modal correspondences across taste, vision, audition, smell, touch and movement. Findings are then discussed in the context of a few theoretical frameworks and directions for future research are provided. Multi-sensory perception lies at the heart of beingalive and having meaningful interactions with the environment, advancing research in this domain would elucidate the mechanisms underlying conscious experiences and information representation- effectively solving the sensory binding problem. Applications of this research range from devising sensory substitution remedies for learning problems in impaired people to optimizing marketing strategies and building a psychological theory of art to experiencing exquisite dining.

The idea that there is some link between all our sensory organs is not alien. The skin parchment illusion demonstrates that there is cross talk between sensory organs and there is feedback from the cross-talk that we perceive. In the experiment demonstrating the illusion, subjects rubbed their hands together and the sound produced was recorded. Replaying the sound to the subjects evoked sensations of roughness as though the skin turned into parchment paper and altering the high frequencies of the sound produced amplified the rough sensation [6].

The Kiki-Bouba effect has been observed across various age groups including infants and cultures from around the world and extensive data reliably validates the effect. Additionally, the effect is observed across multiple types of stimuli including wine, music, foods, walking, drawings, etc. suggesting a cross-modal ubiquity. The putative scientific standpoint is that this phenomenon is universal [7].

\section{TYPICAL DESIGN OF A KIKI-BOUBA EXPERIMENT}

Two shapes are presented to human subjects. One shape is a rounded, bulbous closed figure and the other shape is a jagged, pointy closed figure. The subject is then presented with 2 names which are non-sense words and is asked to match each of the shape with a word. There is generally an agreement of $90 \%-95 \%$ between people over which shape is which word. A number of experiments have tested various groups of people. A variety of non-sense words such as Takete, Kiriki, Teziki, Kichiki and Kekiti have been used as substitutes and in addition to 'Kiki' and words like Maluma, Lomoro, Mamima, Muromu and Malomu for 'Bouba' [8].

\section{The Kiki-Bouba Shapes}

The star-like, edgy \& jagged shape on the left is 'Kiki' and the bulbous, rounded \& smooth shape is Bouba'.

Figure 1 - The Kiki-Bouba shapes
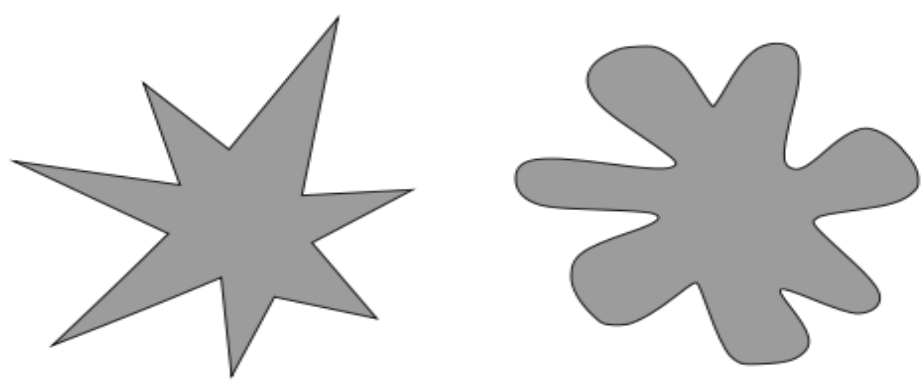
A common characteristic in these experiments is that some employ a congruence versus incongruence aspect to measure cognitive and behavioural task such as discrimination and reaction time. Congruence occurs when the 2 features of a stimuli or a pair of 2 stimuli are both either inherently Kiki or inherently Bouba. Incongruence occurs when the 2 features of a stimuli or a pair of 2 stimuli differ in their KikiBouba-ness.

Table 1 - Congruence and Incongruence with Kiki \&Bouba

\begin{tabular}{|lll|} 
& Kiki [Stimuli 1/feature 1] & Bouba [Stimuli 1/feature 1] \\
\hline Kiki [Stimuli 2/feature 2] & Congruent & Incongruent \\
\hline Bouba [Stimuli 2/feature 2] & Incongruent & Congruent \\
\hline
\end{tabular}

\section{THEORETICAL FRAMEWORKS TO UNDERSTAND THE KIKI-BOUBA EFFECT}

Three theoretical frameworks offer an explanation to bring together various findings with the Kiki-Bouba paradigm. The definitions are given below. The following frameworks help identify and understand the clockwork of cross-modal correspondence.

\section{Sound Symbolism}

Sound symbolism is the idea that sounds naturally carry meaning independent of semantics excluding onomatopoeias, and this becomes one of the precursors to the development of language [9]. Congruence between a sound and a certain features of a referent would make that sound more likely to be used in naming that referent. This characteristic called 'iconicity' is largely implicated in giving people the ability to make 'Kiki-Bouba' discrimination. It is evident that sound-symbolism is a key design feature of the human language [10]. A caveat in how language is acquired lies in the gap in understanding of how seemingly arbitrary sounds are assigned to referents and how non-arbitrary they are in reality, as evidence suggests.

\section{Synesthesia}

Synesthesia is a condition where people reliably, automatically and consistently experience atypical sensory responses in addition to regular \& typical responses for certain sensory inputs [11-12]. For example, a synesthete may see each individual numerical digit as a unique colour involuntarily while fully espousing the meaning of numbers as normal people do. In effect, synaesthesia is co-perceiving of senses. A large body of research has shown that synaesthetic experiences are commonplace in perception and can shed light on how normal humans integrate perceptual experiences [13].

\section{Ideasthesia}

Ideasthesia is defined in the light of linking concepts and perception. In Ideasthesia, the activation of a concept (inducer) evokes a perception like phenomenon (concurrent) [14]. Ideasthesia involves the mediation of cognitive resources in linking the inducers and concurrents. Ideasthesia is a broader phenomenon happening across the senses that explains and widens the scope of synaesthesia-like observations. Synesthesia fails to explain the cognitive/semantic aspect of perception and many synesthetic experiences are, in fact, a manifestation of ideasthesia through some semantic link [15-16]. The Kiki-Bouba effect is suspected to be a prime example of ideasthesia [17]. In ideasthesia, an inducer is semantic in nature and the concurrent is sensory whereas in synaesthesia both are considered to be sensory. For example, thinking of the blue colour as peaceful is ideasthesia whereas seeing blue due to specific words of a peaceful prayer is synaesthesia. The entanglement of biology, concept formation and sensory perception is evident through the Kiki-Bouba paradigm. 
Table 2 - Key publications influencing the growth of the scientific literature on the Kiki-Bouba phenomenon

\begin{tabular}{|c|c|c|c|}
\hline Researchers & Year & Key features & Findings \\
\hline Wolfgang Kohler & 1929 & $\begin{array}{l}\text { Non-sense words: Taketa } \\
\text { \&Baluba } \\
\text { Visual presented shapes: } \\
\text { Jagged and rounded }\end{array}$ & $\begin{array}{l}\text { First evidence of a non- } \\
\text { arbitrary mapping between } \\
\text { non-sense words and shapes }\end{array}$ \\
\hline Wolfgang Kohler & 1947 & $\begin{array}{l}\text { Non-sense words: Taketa } \\
\text { \&Maluba } \\
\text { Visual presented shapes: } \\
\text { Jagged and rounded }\end{array}$ & $\begin{array}{l}\text { Previous study results } \\
\text { replicated and implication } \\
\text { toward a preference of word - } \\
\text { shape association }\end{array}$ \\
\hline $\begin{array}{l}\text { V. S. Ramachandran \& } \\
\text { Edward Hubbard }\end{array}$ & 2001 & $\begin{array}{l}\text { American college students } \\
\text { and Native Tamil speakers } \\
\text { were tested in the traditional } \\
\text { shape sound preference } \\
\text { paradigm }\end{array}$ & $\begin{array}{l}95 \% \text { to } 98 \% \text { showed a } \\
\text { preference of jagged shapes } \\
\text { mapped to Kiki and rounded } \\
\text { shapes mapped to Bouba }\end{array}$ \\
\hline $\begin{array}{c}\text { Daphney Maurer, } \\
\text { Thanujeni Pathman \& } \\
\text { Catherine J. Mondloch }\end{array}$ & 2006 & $\begin{array}{l}\text { Toddlers }(<2.5 \text { years of age }) \\
\text { were tested with the } \\
\text { traditional shape sound } \\
\text { preference paradigm }\end{array}$ & $\begin{array}{l}\text { The associations of Kiki to } \\
\text { jagged shapes and Bouba to } \\
\text { rounded shapes are consistent } \\
\text { even prior to language } \\
\text { development }\end{array}$ \\
\hline $\begin{array}{l}\text { L M. Oberman\&V. S. } \\
\text { Ramachandran }\end{array}$ & 2008 & Autistic children were tested & $\begin{array}{l}\text { Low accuracy with Kiki- } \\
\text { Bouba associations suggest } \\
\text { that there is multi-sensory } \\
\text { integration deficits in autism }\end{array}$ \\
\hline David Barton & 2016 & $\begin{array}{l}\text { Congruent and incongruent } \\
\text { faces tested on affective } \\
\text { responses }\end{array}$ & $\begin{array}{l}\text { Kiki-Bouba is observed in a } \\
\text { social context and has an } \\
\text { affective valence }\end{array}$ \\
\hline
\end{tabular}

\section{KIKI-BOUBA AND SOUND SYMBOLISM}

Two and a half year old children and adults performed similarly on a non-sense word and shape matching task suggesting that having such a natural bias may underlie language development [18]. There is growing evidence that infants have a kernel of higher cognitive functions that enable them to make cross-modal associations and aid the acquisition of language [19-20]. The sound symbolism bootstrapping hypothesis states that sound symbolism is a function of the biological endowment to make sense of multi-modal input which further helps associating sound to unfamiliar objects in a non-arbitrary way. This creates meaning and develops a lexicon to form the inchoate stage in language development [21]. A sound symbolic association between first names and vague descriptions of personality traits was observed by mapping people's silhouettes and names to Kiki or Bouba. Moreover, femininity was found to be associated with Bouba and masculinity with Kiki [22].

A synesthetic bootstrapping hypothesis to describe a model of language development in infancy gains support from Kiki-Bouba experiments flagging visual and auditory signals from the inferior temporal lobe and the auditory cortex respectively with a plausible mediation by the angular gyrus [3]. This offers evidence to support the sound symbolism bootstrapping hypothesis.

A majority of subjects who mapped Kiki-Bouba shapes to a variety of bipolar pairs of words ranging from opposites like yin \& yang to cartoon characters like Astrix and Obelix and from physical characteristics like fat vs. thin to concepts like foolishness vs. intelligence showed mutual agreement on their choices. Owing to the variety of tested stimuli which semantically differ, it is implicated that the correspondence emerges from semantics [17]. 
Intra-modal associations of shape and colour did not parallel a Kiki-Bouba distinction in an internet based experimental study. A number of new words were tested across linguistic borders from all over the world via internet based testing and the results suggest that purely semantic mediation underscores ad hoc inconsistencies [23]. If boulders are Bouba, and Bouba is rated as good and active, how is Bouba also matched to sad emotions for example.

\section{KIKI-BOUBA - EYES HEAR AND EARS SEE THE SAME THING}

Audio-visual presentation of information is commonplace in the digital age. Sounds, lighting and colours are factors that constitute a large portion of audio-visual presentations. One study found that soft musical timbres were associated with Bouba and the colours green, light gray and blue. While, harsh timbres were associated with Kiki and the colours yellow, red and dark gray. There was no association between fundamental frequencies and Kiki-Bouba or fundamental frequencies and colour [24].Researchers showed that even 4-year old children are capable of understanding metaphor and can create audio-visual correspondences which are observed consistently. A high pitch - high volume - bright light and low pitchlow volume-dim light correspondence was demonstrated [25]. In a series of experiments, low/high pitched sounds, bright/dim lights and light/dark colours were used as primary stimuli and accompanying stimuli interchangeably. Subjects were tested on matching pairs of stimuli and were measured on a stimuli discrimination tasks. As hypothesized, subjects performed better when two unimodal stimuli were presented congruently and worse when presented incongruently [26].

\section{KIKI-BOUBA AND THE HELPING SENSES}

Research shows that sense modalities help each other even though they are not always involved in sensing the stimuli.In a haptic-auditory mapping paradigm, neurologically normal and various visually impaired subjects were given two-dimensional and three-dimensional models of the Kiki-Bouba shapes to touch and then map onto the words 'Kiki' and 'Bouba'. Results showed that visual imagery plays a large role in facilitating this haptic and auditory mapping in the absence of actual visual stimuli as the effects were proportionately less pronounced in visually impaired subjects [27]. Japanese subjects show reliable colour shape associations which are likely developed through early language experiences. In a study that tested deaf and hearing subjects on the same colour-shape associations, researchers found that a functioning auditory system plays a role in strengthening the colour-shape associations [28]. This line of research would lie at the heart of developing sensory substitution remedies.

\section{KIKI-BOUBA ACROSS AFFECT}

An affective component has emerged in multiple studies showing that it in intertwined with various simple and complex sensory inputs. Subjects mapped Symmetrical and asymmetrical variants of the Kiki-Bouba shapes with each having two different numbers of protrusions [fewer and higher] to two word dimensions: pleasant-unpleasant and sweet-sour. The results showed that subjects preferred to map symmetrical, Bouba shapes with fewer protrusions as pleasant and sweet; and higher protrusions in asymmetrical Kiki shapes as unpleasant and sour [29]. The role of taste intensity and taste hedonics was investigated with respect to taste-shape correspondence and the results showed that perceived intensity and liking a taste predicted its Kiki-ness and Bouba-ness [30].

Affective congruence was tested on politicians and civilian to investigate a Kiki-Bouba effect between faces and their names. Results showed that there is a preference for faces with congruent sounding names and vice-versa. Moreover, likeability of a certain face facilitates congruency in naming it [31]. Schematic drawings of happy and sad emotions in faces where the mouth took angular and curvy forms was tested with the Kiki-Bouba sound-shape paradigm. Subjects were asked to match non-sense words which were inherently Kiki or Bouba with the emotion-faces. Results showed that matching happy and sad emotions to the appropriate facial expression was in fact observed irrespective of the angular or curvy form of the mouth. It is hypothesized that an internal simulation of the faces and oral gestures dominated the correctness of Kiki vowel sounds to angular form and Bouba to the curvy form [32]. 
With the motivation of understanding consumer behaviour, researchers asked subjects to map tactile attributes of multiple day to day objects like cotton, satin, tinfoil, sandpaper, and abrasive sponge to Kiki and Bouba along with mapping them to adjectives like ugly vs. beautiful and various emotion words. They found that smooth textures like satin are Bouba and sandpaper is Kiki and each object carries some emotional valence [33]. These findings suggest that affect, which is fundamentally not a sensory input, can also be abstracted and mapped as congruent or incongruent with cross-modal associations. One can hypothesize whether a deeper level of abstraction is at play or an entirely different process is involved in enabling an affective component to correspond with sensory data.

\section{KIKI-BOUBA AND TASTE}

Studying the cross modal association between taste, smell and visual aspects of food suggest that typical responses of connoisseurs such as 'The wine is rounded' or 'the taste of a coffee brew is sharp' is in fact grounded in the ability to abstract and perceive through non-stimulated senses [34]. The plate shape and serving presentation of a complex food dish along with their perceived taste and name were used as variables to closely resemble complex dining experiences. Researchers found that there is a sizeable effect of visual cues from food presentation on perceived taste. The apparent sweetness of the dish was affected by the interaction of the plate shape and food presentation. Congruence of plate shape and food presentation resulted in higher ratings for the pleasantness of the name of the food item [35].

A thorough review on taste-shape symbolism for food and beverages show that the following items can be mapped to angular shapes and rounded shapes:

Angular [Kiki-like]: Cranberry jam, Mint chocolate, Salt and vinegar crisps, Dark chocolate, Sparkling water, Maltesers, Cranberry Juice, Tunworth Cheese, Keen's Cheddar and many other bitter tastes.

Rounded [Bouba-like]:Blueberry jam, Brie, Regular crisps, Milk chocolate, Still water, Rolos chocolates, Milk chocolate, Mint Fondant, Lancashire Chedder, Stawley goat's cheese, Leipajuusto cheese and other sweet taste [36-37].

The review also concludes that the shapes seen in food presentation, packaging, sale, etc. determine the hedonic response toward it. An important characteristic of packaging and presentation is the typeface used in it. In a pilot study, taste and typeface (fonts) were used as stimuli and subjects classified sweet as round and 'salty',' sour' and 'bitter' as angular. Additionally, the tastes were mapped congruently with typefacesKiki tastes to Kiki typefaces and Bouba tastes to Bouba typefaces. A follow-up experiment showed that these round/Bouba typefaces were more liked and easier to read than angular/Kiki typefaces. The study concluded that there is evident cross-modal mapping and affective correspondence between taste and typeface [38].

\section{KIKI-BOUBA ACROSS CULTURES}

English Speakers and Tamil Speakers did not show any difference in their tendency to demonstrate the Kiki-Bouba effect [3]. Regardless of a group's proficiency in a particular language, this effect has demonstrated its ubiquity.

In one of the first few hallmark studies, native children from the Mahale peninsula of Tanganyika in Africa were asked to match names to drawings similar to theKiki\&Bouba shapes andwere then compared with English school students. Both subject groups gave highly consistent and matching responses suggesting that the similarities between structural properties of the sound of the names and physical properties of the drawing are acknowledged at an abstract level that underlie naming of objects [39].Another cross-cultural study with Tiawanese and North American subjects revealed finer details about the non-arbitrary matching of radial frequencies with the Kiki-Bouba words. The frequency, amplitude and spikiness of radial frequencies were manipulated and results were consistent with past research with an additional finding that the western subjects weighed amplitude more than their eastern counterparts and weighed spikiness lesser suggesting that the responses were partially influenced by culture based perceptual experiences [40]. Subjects from the Himba population of Northern Namibia, a culture isolated from western influence showed shape-sound symbolism similar to that in the westerners but differed in shape-taste mapping. In 
contrast with the westerners, the Himba did not map aerated sparkling water to angular shapes and in fact, mapped less bitter milk chocolates to angular shapes. The study suggests that there is a very strong influence of culture, environment and phylogenetics on cross-modal mapping and is not purely universal between any two sense modalities [41].

\section{KIKI-BOUBA AND BODY PHYSICS}

Not much research is done with respect to physical movements. Walking motions were studied with respect to a Kiki and Bouba differentiation. Researchers found that a high velocity, angular 'shaky' walk mapped to Kiki and a low velocity, smooth and continuous walk was mapped to Bouba [42]. The study suggests that muscle movements alongside the feedback from the associated imagery contributes to this cross-modal association. Similar conclusions can be drawn from studies involving vocalizing sounds. Articulatory properties of the words and the mouth movements to produce such sounds are implicated in making the Kiki-Bouba association alongside the actual physical structure of the written word. A study found that both influence the shape-sound mapping with the highlight of literate subjects demonstrating a stronger influence from the grapheme as compared to the purely auditory form of the word [43]. In a bottom up task to build novel words to label abstract shapes, the effects of both visuomotor effects of vowel form and perceptive qualities of the auditory form play a role in labelling. Furthermore, a strong parity is formed between plosive consonants, non-rounded vowels and jagged shapes as well as parity between sonorant consonants, rounded vowels and curvy shapes limited within words that are easy to articulate [8].

\section{KIKI-BOUBA AND BIOLOGY}

The biological basis of this phenomenon is not explicitly tested. Nonetheless, certain findings, on facevalue, correspond with implicit theoretical constructs.In an experiment controlling for sensory inputs in cats via uni-modal and multi-modal stimuli, Auditory, Visual and Somatosensory signals from the anterior ectosylvian sulcus and the visual signals from the lateral sylvian were found to converge at the multisensory neurons' site in the superior colliculus. This study shows that the relationship between a specific senses' cortical areas and the corresponding neurons in the superior colliculus is strong. Moreover, the unimodal presentation of sensory inputs aided the identification of individual locations to map the corresponding neural activity. Results from this study indicate that the output neurons of the superior colliculus have strong cortical control and thus have a powerful influence on cognition and behaviour [44]. In an extensive review paper, multisensory integration with respect to the auditory sense was discussed. The literature suggests that the neural signals from the auditory pathway are significantly modulated by the visual system and the somatosensory system. Additional investigations showed that this modulation occurs at a minimum of three different locations in the ascending auditory pathway: Inferior colliculus, Medial Geniculate Body and Auditory Cortex [45].

A hallmark investigation concurring with past research showed that Cross-modal mapping of low luminescence to low pitch sounds and high luminescence to high pitch sounds may have evolved before humans. Chimpanzees performed similar to humans. This suggests that such an ideasthetic phenomenon is ingrained in primate sensory systems. Ideasthetically congruent stimuli make task performance easier in both humans and pan-troglodytes [46]. Researchers, for the first time, employed a decision making task with fMRI imaging where participants classified congruent and incongruent non-sense word-shape combinations under time pressure. Their investigation showed that incongruent pairs elicited higher activation in the frontal cortices which plausibly reflected the incongruence itself. One can hypothesize that incongruence utilized more cognitive resources. Furthermore, they found a difference in behavioural sensitivity index correlated with the lateral occipital cortex indicating that subjects that were sensitive to unimodal visual stimuli were also more sensitive to shape-sound stimuli. There appears to be an effect of dominance between sense modalities [47].

Dysfunctional mirror neurons are implicated in Autism. Research has also shown that autistic children cannot fathom metaphor and tend to think literally [48]. The Kiki-Bouba effect becomes in valid and 
unreliable with autistics with a just-above-chance consistency amongst subjects (as compared to more than $90 \%$ in normal people) [5]. A mirror neuron based theory of abstraction suggests that there is a close association with physical movement and the experience/object that requires abstraction. It is speculated that the function of copying gestures and creating metaphor became more sophisticated and formed connecting links to various other sensory modalities with newer evolved functions, thus granting the ability to abstract between sensory modalities [49]. This body of research shows that there are a number of neural substrates that generate hypotheses for cross-modal abstraction.

\section{ANALYSES AND DISCUSSION}

A large body of research involving Kiki and Bouba is associated with Sound Symbolism, Synesthesia and Ideasthesia [4]. This phenomenon underlies mechanism(s) that either create an association between stimuli and/or identify a common denominator. It is glaringly evident that humans can extract commonalities between seemingly unrelated objects and experiences. It is also conclusively visible that there is nonarbitrary mapping between stimuli features. However, studies cited in this paper largely focus on the description and the phenomenological aspects of the predominantly non-arbitrary cross-modal mapping but not the process in which such an abstraction is formed at the biological and cognitive level.

\section{Language development and sound symbolism}

In a classic Kiki-Bouba paradigm, researchers presented words and syllables to Native Tamil speakers and found out that Kiki-Bouba phenomenon is clearly observed in other languages [3]. This phenomenon is also observed in non-English children. It could thus be hypothesized that the abstraction ability precedes language development and supports concept formation and meaning assigning through naïve sensations [20-21].The evolution of language may have its roots in this non-arbitrary association of sounds and objects. This association is well described by sound symbolism and may have led to an inherent preference of certain sounds for certain objects in the environment.

\section{Source of abstraction in sound-shape mapping}

There is growing evidence to suggest that it is not only the superficial and obvious features of the shape that match the words in shape-sound symbolism. The audio signals, in their waveform, associated with pronouncing the words also match with respect to having a dominating 'rounded' waveform for Bouba and a 'jagged' form for Kiki. Moreover, actual movement of the mouth to say 'Bouba' corresponds with a round shape and the movement for 'Kiki' is broken, repetitive and strained [43]. It is unclear at this stage if the mouth movement, the auditory form, the perceived sound of the words or the grapheme contribute for this abstraction. It is suspected that all may contribute. Although, there is growing evidence to implicate the mirror neuron system as it offers to explain the mouth movement to shape correspondence.

\section{Congruence versus incongruence}

The evidence points to one principle in affective evaluations and cognitive tasks. Congruence leads to a favourable affective evaluation and it also aids in cognitive tasks like classification [24,31]. Congruence would therefore influence, in part, the daily social interactions and preference of people in authority. Congruence would also influence aspects of learning under time pressure and facilitate concept formation through interpreting a 'web' of congruent aspects. Whereas, incongruence would induce a feeling ranging from, 'dislike' to 'unsettling'. As the body of literature grows with respect to affect and congruence, the clock-work behind complex social behaviours and how people make rapid decisions about preferences would become clearer.

As seen in multiple studies, Kiki and Bouba are used as a Stimulus as well as a response. Assuming that the stimuli and response are completely interchangeable to create non-arbitrary cross-modal associations, one can suspect that this common denominator that facilitates the associations is an emergent phenomenon, at least in part. It is unclear if the neural circuitry can function normally and still not facilitate such abstraction. 


\section{Kiki Bouba in Art}

Composition in painting, thought behind music and sculpting, the perfect blend of cocktails and cuisines, etc. are popularly considered as artistic processes. It may not be a naïve statement to consider that crossmodal correspondences lie at the heart of speaking metaphor and drawing parallels between a moment of inspiration and its representation as art. Art is hard to understand and define because of it lacking objectivity, among many other things. The Kiki-Bouba paradigm exhibits many cross-modal pairs that are pleasant (or unpleasant) due to congruence (or incongruence). It can be conjectured that such choices underlie artistic thought.

\section{Cross modal mapping without using Kiki-Bouba}

There are cross-modal research studies that do not use the Kiki-Bouba paradigm but nonetheless have a similar mapping/association protocol. For example, in one study people tried to map angles between two lines to colours [50]. However, a proposed match between colour and shape has yielded inconsistencies in replication studies using the Implicit Association Test [51]. In another study, certain tastes were mapped to different pitches of sounds [52]. Researchers also incorporated a subjective evaluation of taste and sound in a study where professional musicians improvised based on mapping the sound onto taste words. In a follow-up experiment, untrained listeners classified the improvisations based on what taste they should elicit [53]. In all 3 studies (amongst many more), the results are significant and non-random.

\section{A proposed explanation -1}

Based on the gathered data building a complete model of how abstract associations work is far from materializing. Nonetheless, certain inferences can be made. The hub \& spoke model of synaesthesia lays the groundwork for a strong framework to explain cross-sensory mapping and semantic entanglement with different experiences. As per this model, the inducer and concurrent at a psychophysical and neural level 'talk' with each other at supra-modal 'hubs'. These hubs relay information to a distributed network of cortical areas which engender the subjective experience of perception via 'spokes' that extend around the network [16]. It can be hypothesized that one of these hubs is the multi-sensory neuron area of the Superior Colliculus.

\section{A proposed explanation -2}

As far as current research goes, ideasthesia explains abstraction and non-arbitrary associations quite robustly. Hypotheses involving sound symbolism explain language development with strong evidence but do not contribute to the Kiki-Bouba effect with non-auditory cross-modal associations. Symbolism may be appended to other sensory modalities (touch-symbolism, taste-symbolism, etc.) and these may become theoretical factors in future models. The myriad cross-modal mappings and Kiki-Bouba associations suggest that the paradigm elicits intrinsic features of ideasthesia and forward the development of a unified, all-encompassing theory of sensation \& perception, and effectively solve the binding problem.

Previously, cross-modal binding seemed to be a result of spatio-temporal markers [4]. Today, in addition to spatio-temporal markers, cross-modal binding is in part due to an ideasthetic abstraction and nonarbitrary mapping between experiences. People's veracity of cross-modal mapping and a very reliable KikiBouba effect across a plethora of stimuli shows that a theory that explains this must incorporate the biological connections between neurons as well as a complex web of cognitive links between percepts as well as account for a mental faculty that permits abstracting novel stimuli by means of updating stimuli representations.

\section{LIMITATIONS AND FUTURE DIRECTIONS}

1. A design flaw:Most studies use a paradigm which forces subjects to contrast with 2 shapes. Few studies employ a continuum from Kiki to Bouba. Use of such a continuum would likely map the complexity of stimuli in more comprehensive ways. It would also remove the forced-choice pressure on subjects to map one stimuli to Kiki and ergo, for lack of options, the other stimuli to Bouba. This may dissolve the ad hoc inconsistencies seen with the semantic mediation hypothesis. 
2. 2 Dimensional and 3 imensional Kiki-Bouba:Another aspect of the Kiki-Bouba spectrum is that most studies include a bifurcation of $2 \mathrm{D}$ imagery or $3 \mathrm{D}$ imagery but not both. A mixture of $2 \mathrm{D} \&$ $3 \mathrm{D}$ shape presentation would likely indicate if the abstract mapping has a third independent factor of 'depth'. Musicians and Visual artists often indicate that some artistic content lacks depth and appears superficial and vice versa. In light of developing a robust model to describe cross modal cross talk, one would need to account for as many common denominators as there can be which can be supported by evidence as in the case of Kiki and Bouba. Although, many studies do employ 3 shapes: Kiki, Kiki-Bouba mixed and Bouba.

3. Relativity: Current literature does not take into account relativity between stimuli. This is a likely area of erroneous findings. One visual aspect may be Bouba in comparison to a very strong Kiki but yet be Kiki in front of a softer aspect. Comparing three degrees of one stimuli or three independent stimuli would yield more sensitive results and address the question of whether Kiki and Bouba remain absolute or are the effects heavily context dependent. Consequently, adopting a continuum for the Kiki-Bouba spectrum will yield more comprehensive results.

4. The emergence of the effect: It is unclear whether the least common denominator between any two[or more] stimuli is responsible for this effect. It is also unclear whether there is any least common denominator in the first place or such a denominator is created as an emergent phenomenon. As per the gestalt school of thought, the whole is greater than the sum of its components. While tethering the idea of abstract cross-modal correspondences to this notion, is it possible that the facilitator of the whole gestalt is this very emergent phenomenon? Although this remains at the level of speculation, future studies can address this question. Another question that arises is whether this, presumably, emergent phenomenon serves evolutionary advantages as it is observed in pan-troglodytes and regions analogous to 'hubs' are identified in cats. Future studies could focus on identifying the cognitive and biological conditions that are needed to enable the experience of this effect.

5. Intra-stimuli competition: Most studies can be put in the category of 'classifiers'. Subjects map the given stimuli to its abstract representation (shape, word, taste, sound, etc.). Choosing to present the same stimuli across multiple sensory modalities and mapping the stimuli on a spectrum while controlling for other senses will shed light on different aspects of the same stimuli. Consequently, it will reveal which of the two sensory modalities of the stimuli can be considered a Kiki whilst the other a Bouba(and vice-versa). A tall round mug with hot coffee in it maybe visually a Kiki but touching it and tasting the coffee may be a Bouba, for example. In such a situation, can it be objectively measured? If yes, how would the gestalt of the stimuli be? Would each sensory modality abstract uniquely or will a compound and complex abstraction be made. Does a specific sensory input dominate or each one is 'weighted' by the brain to form the ideasthetic concurrent to further form an abstraction for drinking coffee with that specific mug?

6. Inter-stimuli competition: Although this paradigm is largely used in the context of cross-modal correspondences, the effect between competing features within a single modality or 2 features in 1 modality versus 3 features in another modality is relatively unexplored. Competing stimuli, once factored in, would represent day-to-day interactions better and provide a ground for higher yield in the consumer market.

7. Clinical applications: A number of novel ways to teach concepts to children can be constructed once the interaction between all senses and the semantic nature of a concept is understood. This will effectively bolster learning in relatively weaker students and perhaps help mentally retarded, autistic and disabled people acquire abstract learning skills. Future research can be conducted with respect to utilizing congruence and incongruence along with multi-modal scaffolds (for example, use touch as a mode to acquire information that inherently has audio and visual features) as a teaching methodology and therapy. 
8. Consumer and job market: In a world where digital projections of products and ideas flourish, the consumer passes quick judgments and makes decisions on whether to indulge in a particular product or idea. Marketing strategies can reap benefits from how consumers behave with the mediation of cross-modal correspondences and evaluations. For example, the reactions people have to a product may be due to its design that elicits a very strong Kiki response that conflicts with its function that elicits a Bouba response. Imagine a tooth-brush which is meant to be very soft looks like its bristles can cut:an angry tooth-brush. The applications of cross-modal correspondences in product design are endless. Furthermore, humans as resources undergo extensive training in soft-skills. Research from this field would likely add more theoretical grounding in body-language analysis and manipulation of appearances to suit a particular situation.

9. Sensory Substitution: Kiki-Bouba experiments have in principle opened the gate for developing ways in which blind people can see art or deaf people can learn music. As observed in the current findings, the Kiki-Bouba shapes and words have been interchangeably used as stimuli as well as responses. The interchangeability and assistance of senses in concept formation would likely spearhead the development of sensory substitution techniques.

10. Kiki-Bouba in the clinical setting:One primary deficit observed in the body of literature is the lack of clinical populations being tested. We are still in the dark when it comes to understanding the response patterns in patients with personality disorders, psychosis and anxiety disorders. This begs the question, 'would incongruence in a multi-sensory stimulus induce anxiety or discomfort?'. An extension of this problem would be to understand pet behaviour. Would children and animal reactions be contingent on the abstract qualities of objects in their environment? These questions would make research in this field a priority when it comes to designing rehabilitation centres, pet homes, houses, work-places and care-facilities for patients.A question that is open to answering is whether familiarity with a certain stimuli changes its Kiki-ness or Bouba-ness over time. This question also extends toward changes in emotional valence, anxiety reactions, etc. to the stimuli. In the event that Kiki and Bouba are highly relative to each other and changeable across time, could it have use in treating mental disorders?

11. Classroom training:Lastly, a proposed direction in utilizing this research and furthering the literature itself is the class-room teaching setting where academic concepts can be tested for their multi-modal presentation. Complex concepts typically take time to be thoroughly encoded and espoused by students. Shortening the time invested in learning while reducing the rate at which conceptual learning is blurred/forgotten would subsequently raise the standard of education for all types of people in all types of settings.

\section{CONCLUSIONS}

This review discusses the existing literature associated with the Kiki-Bouba effect- tendency of people to name a jagged star-like shape 'Kiki' and a round bulbous shape 'Bouba'. The existing literature is dense in its description of the phenomenon but limited when it comes to explaining the underlying mechanisms and origins. The study of this effect will eventually unsheathe the mechanisms for sensory-binding. In addition to temporal and spatial factors of defining the sensory data, abstract correspondences in ideasthesia bring us closer to understanding the sensation-perception nexus. The effect is universal and generalizable.Future research directions include sensory substitution mechanisms, understanding consumer behaviour, building a model of cross-modal sensory integration and inventing novel teaching methods among many others.

\section{REFERENCES}

1. Kohler W. Gestalt Psychology. New York : Liveright: 1929.

2. Kohler W. Gestalt Psychology: An Introduction to new concepts in Modern Psychology. New York: Liveright; 1947. 
3. Ramachandran VS, Hubbard EM. Synaesthesia - a window into perception, thought and language. J Consc Stud 2001;8:3-34.

4. Spence C. Cross-modal correspondences: A tutorial review. Attention, Perception and Psychophysics 2011;73(4):971-95.

5. Oberman, LM, Ramachandran VS.Preliminary evidence for deficits in multisensory integration in autism spectrum disorders: The mirror neuron hypothesis. Soc Neurosci 2008;3(3-4):348-55.

6. Jousmaki V, Hari R.Parchment-skin illusion: sound-biased touch. Curr Biol 1998;8(6):190-6.

7. Ramachandran, VS, Hubbard EM. The emergence of the Human mind: Some clues from synesthesia. In: Robertson LC, Sagiv N.(eds). Synesthesia: Perspectives from neuroscience. Oxford University Press; 2004. pp.147-90.

8. Nielsen AK, Rendall D.Parsing the role of consonants versus vowels in the classic Takete-Maluma phenomenon.Can J Exp Psychol 2013;67(2):153-63.

9. Lockwood G, Dingemanse M. Iconicity in the lab: a review of behavioral, developmental, and neuroimaging research into sound-symbolism. Front Psychol 2015;6:1624.

10. Hocket C. The origin of speech. Sci Am 1960;203:89-97.

11. Lupiáñez J, CallejasA. Automatic perception and synaesthesia: evidence from colour and photism naming in a stroop-negative priming task.Cortex 2006;42(2):204-12.

12. Mylopoulos, MI, RoT. Synesthesia: a colorful word with a touching sound?. Front Psychol 2013;4:763.

13. Cytowic RE, Eagleman D. In. Wednesday Is Indigo Blue: Discovering the Brain of Synesthesia.. Cambridge, MA: MIT Press; 2009.

14. Chiou R, Rich AN.The role of conceptual knowledge in understanding synaesthesia: Evaluating contemporary findings from a "hub-and-spokes" perspective. Front Psychol 2014;5;105.

15. Dixon MJ, Smilek D, DuffyPL, Zanna PM, Merikle PM.The Role of Meaning in Grapheme-Colour Synaesthesia. Cortex 2006;42:243-52.

16. Mroczko-WąsowiczA, Nikolić D.Semantic mechanisms may be responsible for developing synesthesia. Front Neurosci 2014;8:509

17. Emilio G, Oscar I, María J, Juárez-Ramos V, Rodríguez A, José L. The Kiki-Bouba Effect A Case of Personification and Ideaesthesia. J Consc Stud 2013;20(1-2):84-102.

18. Maurer D, Pathman T, Mondloch CJ. The shape of boubas: sound-shape correspondences in toddlers and adults. Development Sci 2016;9:316-22.

19. Andrew NM. Towards a developmental cognitive science. The implications of cross-modal matching and imitation for the development of representation and memory in infancy. Ann NY Acad Sci 1990;10:608-20.

20. Ozturk O, Krehm M,Vouloumanos A.Sound symbolism in infancy: evidence for sound-shape cross-modal correspondences in 4-month-olds. J Exp Child Psychol 2013;114(2):173-86.

21. Matsumi I, Sotaro K. The sound symbolism bootstrapping hypothesis for language acquisition and language evolution.Philos Trans R Soc Lond B Biol Sci 2014;369(1651):20130298.

22. Sidhu, DM, Pexman PM.What's in a Name? Sound Symbolism and Gender in First Names. PLoS one 2015;10(5):e0126809.

23. Woods AT, Specnce C, Butcher N, Deroy O. Fast lemons and sour boulders: Testing crossmodal correspondences using an internet-based testing methodology. Iperception 2013;4(6):365-79.

24. Adeli M, Rouat J, Molotchnikoff S. Audiovisual correspondence between musical timbre and visual shapes. Front Hum Neurosci 2014;8:252.

25. Marks LE, Hammeal RJ, Bornstein HM.Perceiving similarity and comprehending metaphor. MonogrSoc Res Child Dev 1987;52(1):1-102.

26. Marks LE. On cross-modal similarity: auditory-visual interactions in speeded discrimination. J Exp Psychol Hum Percept Perform 1987;13(3):384-94.

27. Fryer L, Freeman J, Pring L.Touching words is not enough: how visual experience influences hapticauditory associations in the "Bouba-Kiki" effect. Cognition 2014;132(2):164-73.

28. Chen N, Tanaka K, Namatame M, Watanabe K. Colour-shape associations in deaf and hearing people. Front Psychol 2016;7:355.

29. Salgado-Montejo A, Alvarado JA, Velasco C, Salgado CJ, Hasse K, Spence C.The sweetest thing: the influence of angularity, symmetry, and the number of elements on shape-valence and shape-taste matches. Front Psychol 2015;6:1382.

30. Velasco C, Woods AT, Liu J,Spence C.Assessing the Role of Taste Intensity and Hedonics in Taste-Shape Correspondences. Multisensory Research 2016;1(3):209-21.

31. Barton D.Exploring a social "bouba-kiki" effect and the affective consequences of having a congruent versus incongruent name. Thesis, Doctor of Philosophy 2016, University of Otago. 
32. Karthikeyan S, Rammairone B, Ramachandra V.The Bouba-Kiki Phenomenon Tested via Schematic Drawings of Facial Expressions: Further Validation of the Internal Simulation Hypothesis. Iperception 2016; 7(1):(In press).

33. Etzi R, Spence C, Zampini M, Gallace A.When Sandpaper Is 'Kiki' and Satin Is 'Bouba': an Exploration of the Associations Between Words, Emotional States, and the Tactile Attributes of Everyday Materials. Multisensory Research 2016;1(3):133-55.

34. Spence C, Deroy O.On the shape of flavours: A review of 4 hypotheses. Theoria Et Historia Scientiarum 2014;10:207-38.

35. Fairhurst MT, Pritchard D, Opsina D, Deroy O.Bouba-Kiki in the plate: combining crossmodal correspondences to change flavour experience. Flavour 2015;4:22.

36. Spence C, Mary KN.Assessing the shape symbolism of the taste, flavour, and texture of foods and beverages. Flavour 2012;1:12.

37. Gallace A, Boschin E,Spence C. On the taste of "Bouba" and "Kiki": An exploration of word-food associations in neurologically normal participants. Cogn Neurosci 2011;2(1):34-46.

38. Velasco C, Woods, AT, Hyndman S, Spence, C. Taste of typeface. Iperception 2015;6(4):(In Press).

39. Davis R. The fitness of names to drawings. A cross-cultural study in Tanganyika. Br J Psychol 1961;52(3): 259-68.

40. Yi-Chuan C, Pi-Chun H, Andy W, Spence C. When "Bouba" equals "Kiki": Cultural commonalities and cultural differences in sound-shape correspondences. Science Reports 2016;6.

41. Bremner AJ, Caparos S, Davidoff J, de Fockert J, Linnell KJ. Spence, C.Bouba" and "Kiki" in Namibia? A remote culture make similar shape-sound matches, but different shape-taste matches to Westerners. Cognition 2013;126(2):165-72.

42. Koppensteiner M, Pia S, Jäschke JPM.Shaking Takete and Flowing Maluma. Non-Sense Words Are Associated with Motion Patterns. PLoS One 2016;11(3):(In Press).

43. Cuskley C, Simner J, Kirby, S. Phonological and orthographic influences in the bouba-kiki effect. Psychol Res 2015 (Epub ahead of print).

44. Wallace MT,Meredith MA, Stein BE.Converging influences from visual, auditory, and somatosensory cortices onto output neurons of the superior colliculus. J Neurophysiol1993;69(6):1797-1809.

45. Wu C, Stefanescu RA, Martel DT, Shore SE. Listening to another sense: somatosensory integration in the auditory system. Cell Tissue Res 2015;361(1):233-50.

46. Vera UL, Ikuma A, Tetsuro M.Visuoauditory mappings between high luminance and high pitch are shared by chimpanzees (Pan troglodytes) and humans. Proc Natl Acad Sci USA 2011;108(51):20661-5.

47. Nathan P.Exploring the bouba/kiki effect: a behavioral and fMRI study. Service des Maladies Inectieuses et Tropicales, Hôpital Bichat - Claude-Bernard (Hôpitaux Universitaires Paris Nord Val de Seine); 2015.

48. Ramachandran VS, Oberman LM.Broken mirrors: a theory of autism. Sci Am 2006;295(5):92-9.

49. Acharya S, Shukla S.Mirror neurons: Enigma of the metaphysical modular brain. J Nat Sci Biol Med 2012; 3(2):118-24.

50. Albertazzi L, Malfatti M, Canal L, MiccioloR. The hue of angles- was kandinsky right?.Art \& Perception 2015;3(1):81-92.

51. Makin ADJ, Wuerger SM.The IAT shows no evidence for Kandinsky's color-shape associations. Frontiers in Psychology 2013;4:616.

52. Wang Q, Spence C. 'Striking a Sour Note': Assessing the Influence of Consonant and Dissonant Music on Taste Perception. Multisensory Research 2016;1(3):195-208.

53. Mesz B, Trevisan MA, Sigman M. Taste of Music. Perception 2011;40:209-19.

$$
\begin{aligned}
& \text { Acknowledgements - Nil } \\
& \text { Source of Funding - Nil } \\
& \text { Conflict of Interest - Nil }
\end{aligned}
$$

